\title{
Auroras boreales, un fenómeno natural que facilita la enseñanza de la química
}

Rojas Laura'1,2López Oscar1,3 Muñoz Liz'1,4

Categoría 1. Reflexiones y experiencias desde la innovación en el aula.

\section{Resumen}

En el presente artículo se encuentra el desarrollo de una propuesta didáctica, aplicada a los estudiantes de décimo grado del colegio Alfredo Iriarte "sede A". La propuesta tiene como fin explicar conceptos básicos dentro de la química tales como átomo, molécula partículas sub atómicas, entre otros, a partir de un acontecimiento cotidiano, con el que se busca captar la atención de los estudiantes dentro del aula escolar. El fenómeno natural bajo el cual se desarrolló la propuesta es "Auroras Boreales"; Inicialmente se realiza una explicación general del fenómeno, identificando los conceptos previos que poseían acerca de la química, para finalmente, cuales eran los conceptos que se debía enfocar la explicación y así lograr que la temática lograra generar interés y una correcta interiorización por parte de los estudiantes, observado a través de un instrumento de evaluación.

\section{Palabras clave}

Modelos didácticos, fenómenos naturales, auroras boreales.

\section{Introducción}

La pedagogía tradicional surge en el siglo XVI, como una forma de transmitir el conocimiento hacia las siguientes generaciones, basándose en la disciplina y repetición como sus principales pilares y ejes de funcionamiento. Hoy por hoy después de varios siglos de cambios y reformas ajustadas a los factores, económicos, sociales, culturales y políticos de cada sociedad, observamos en nuestras aulas un gran número de oportunidades para el desarrollo de la

'Estudiante Universidad Distrital Francisco José de Caldas

2 Laura_20rojas@hotmail.com

3 Oalopezr10@gmail.com

4 Liz2007@gmail.com 
Revista Tecné, Episteme y Didaxis: TED. Año 2014, Número Extraordinario. ISSN Impreso: 0121-3814, ISSN web: 2323-0126

Memorias, Sexto Congreso Internacional sobre Formación de Profesores de Ciencias. 08 al 10 de octubre de 2014, Bogotá

educación, las cuales se truncan por la falta de ideas e innovación de los docentes a la hora de enseñar.

A partir de lo anterior como estudiantes de Licenciatura en Química se realiza este trabajo con el objeto de explorar el conocimiento previo que poseen los estudiantes acerca de la química, desde un enfoque educativo constructivista, en cuyo marco subyacen modalidades teóricas y prácticas, abordando conceptos y explicaciones químicas desde el fenómeno "Auroras Boreales". Mediante este fenómeno se empezó a explorar y profundizar conceptos como átomo, ion, molécula, electrón, protón y neutrón, partiendo desde un contexto histórico- cultural, hasta describir, analizar y comprender temas como efecto fotoeléctrico, emisión y absorción atómica; Buscando siempre generar en los estudiantes interés por la temática y una apropiación de los conceptos.

\section{Modelos didácticos}

Al transcurrir los años, el desarrollo de conceptos y modelos útiles representa actividad central en la enseñanza de la Química. La utilidad de un concepto o modelo se encuentra en relación directa con su capacidad predictiva de explicación de los fenómenos químicos, (Castro E.A 1990), es por eso que para explicar nuestra temática "Auroras Boreales", se tomó como referencia un modelo constructivista donde se le permitiera a los alumnos construir su propio saber y un modelo tradicionalista en el sentido de explicar claramente y exponer de manera progresiva cada uno de los conceptos, es complicado asumir cada uno de los procesos de aprendizaje tanto experimentales como teóricos e históricos. Aquí la idea principal es la de transposición didáctica (Chevallard, 1997), que indica los procesos por medio de los cuales el conocimiento científico se transforma de manera que sea posible su aprendizaje por los alumnos, independientemente de su edad y de sus condiciones socioculturales.

Para la temática trabajada se manejó un modelo que lograra incentivar a los estudiantes, reconociendo las dificultades sociales que los envolvía, buscando siempre transmitir y enfocar la explicación química del fenómeno "Auroras Boreales".

\section{Modelos y representaciones.}

Para explicar la temática "Auroras Boreales", principalmente se utilizó los modelos atómicos desde un enfoque histórico y su importancia a través del tiempo, gracias al modelo de Bohr se logra explicar la formación de las Auroras Boreales a 
Revista Tecné, Episteme y Didaxis: TED. Año 2014, Número Extraordinario. ISSN Impreso: 0121-3814, ISSN web: 2323-0126

Memorias, Sexto Congreso Internacional sobre Formación de Profesores de Ciencias. 08 al 10 de octubre de 2014, Bogotá

partir de la emisión y absorción atómica, desarrollando así en los estudiantes la capacidad de relacionar e identificar los sucesos macro con la explicación micro, basados en el efecto fotoeléctrico, y la interacción entre radiación y la materia.

Con respecto a las representaciones se empleó para la explicación de las Auroras Boreales las imágenes que se presentan a continuación.

Imagen 1. Formación "Auroras Boreales". Imagen A. El viento solar viajando a través del espacio, pasando por la tierra e interactuando con el campo magnético. Imagen B. Interacción de los electrones del viento solar con las moléculas de nitrógeno y oxígeno contenidos en la ionosfera. Imagen C. Aurora Boreal, vista en los polos geográficos sur y norte respectivamente.
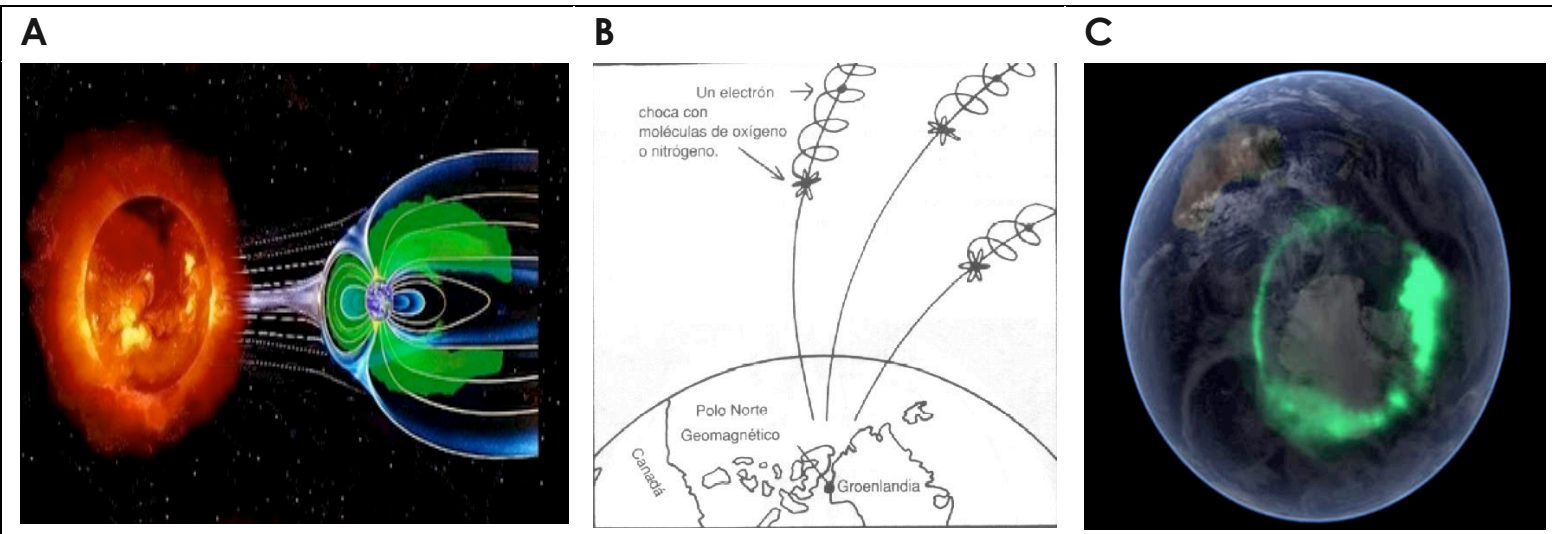

\section{Explicación química del fenómeno}

La formación de las auroras se puede explicar a sabiendas de que el Sol emite continuamente y en todas las direcciones, un flujo de partículas cargadas, flujo que recibe el nombre de plasma. Las partículas de plasma, "guiadas" por el campo magnético del Sol, forma el viento solar que viaja a través del espacio a unos $400 \mathrm{~km} / \mathrm{s}$, llegando a la Tierra en 4 o 5 días, (figura A). En la Tierra gracias a que los polos magnéticos Norte y Sur coinciden con los polos geográficos Sur y Norte, respectivamente. El campo magnético es más intenso donde las líneas de campo están más juntas, es decir en los polos, de manera que, las partículas cargadas que logran entrar en el campo magnético terrestre -la gran mayoría no lo consigue- son reconducidas hacia los polos magnéticos (Grafico A). En su camino de descenso pasan por la ionosfera, que es una capa que limita exteriormente a la atmosfera (a unos $60 \mathrm{~km}$ de altura) y en la que se encuentran muchos iones: átomos de oxígeno y nitrógeno con carga eléctrica, originados por los rayos ultravioleta procedentes del Sol. La ionosfera actúa como 
Revista Tecné, Episteme y Didaxis: TED. Año 2014, Número Extraordinario. ISSN Impreso: 0121-3814, ISSN web: 2323-0126

Memorias, Sexto Congreso Internacional sobre Formación de Profesores de Ciencias. 08 al 10 de octubre de 2014, Bogotá

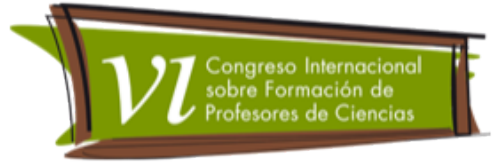

medio conductor para las partículas cargadas que llegan con el viento solar, y es en ella en donde se produce la aurora, entre 90 y $110 \mathrm{~km}$ de altura, (Grafico B)

Los electrones chocan con las moléculas de oxígeno y nitrógeno excitándolas, y estas, luego, se des excitan, emitiendo luz: verde las de oxígeno y roja las de nitrógeno, (Grafico C).

Imagen 2. Representación: Emisión y absorción atómica

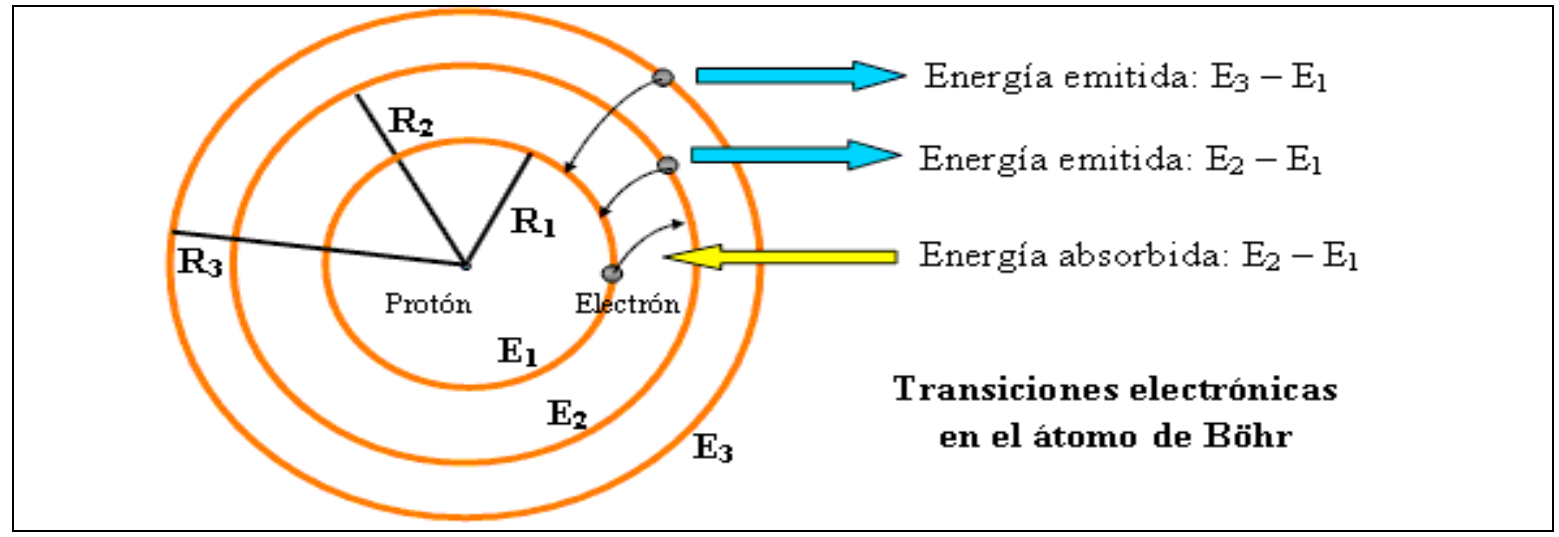

El modelo atómico de Bohr (imagen 2), nos permite dar una explicación micro del fenómeno "Auroras Boreales ".

\section{Marco metodológico}

Las actividades que se desarrollaron con los estudiantes son las siguientes:

Noviembre 2: presentación estudiantes, realización actividad de entrada y explicación de lo que son las auroras boreales.

Noviembre 4: Socialización de conceptos, reseña histórica de los modelos atómicos, explicación química y física de la formación de las auroras boreales a partir de un video didáctico, Este vídeo creado por "forskning.no" en colaboración con el departamento de física de la universidad de Oslo.

Noviembre 9: explicación de fluorescencia. Y evaluación del proceso de aprendizaje.

\section{Resultados y análisis}

Fue de suma importancia iniciar con un discurso llamativo para que poco a poco los estudiantes se fueran involucrando en la clase y fueran dejando ver su 
Revista Tecné, Episteme y Didaxis: TED. Año 2014, Número Extraordinario. ISSN Impreso: 0121-3814, ISSN web: 2323-0126

Memorias, Sexto Congreso Internacional sobre Formación de Profesores de Ciencias. 08 al 10 de octubre de 2014, Bogotá

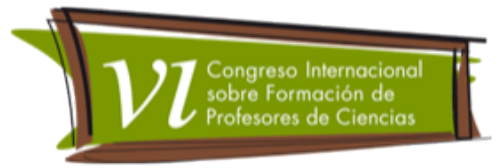

entusiasmo por la temática (auroras boreales). Temática que inicialmente se la presentamos en una lectura acompañada de algunas imágenes (imagen 1) las cuales definitivamente les permitieron conocer las auroras boreales y empezar a cuestionarse del por qué dicho fenómeno.

En la primera clase se aplicó la actividad de entrada (cuestionario "auroras boreales"), como se puede apreciar en la tabla 2, a la primera pregunta pedía que se definiera, que era átomo, los estudiantes contestaban, "el átomo es la partícula más pequeña de la materia", de modo que, para el estudiante, la idea de los orbitales, los números cuánticos, el espín, etc. queda despojada de su "plataforma» metodológica sin la cual se pierde la comprensión meridiana del asunto (Ferro, et al, 1995), pocos enunciaron, los protones, electrones y neutrones y conceptos como núcleo y orbitales; la segunda pregunta que consistía en dibujar el átomo, se observó una visión muy diferente en la cual involucraban la forma, las partículas sub-atómicas, los orbitales, dejando de lado las representaciones cotidianas de esferas y líneas curvas para pasar a núcleo y electrones alrededor de este sin tener visión de orbitas fijas sino de zonas dentro de la cuales podíamos encontrar el electrón (imagen 3); sin embargo de que sabían cuáles eran las partes del átomo, no tenían claro cuál era su ubicación e incluso confundían las cargas de las partículas sub-atómicas (imagen 4); por ello se entiende que los estudiantes tenían memorizado el concepto de átomo, sin entenderlo.

Tabla 2. Resultados de la conducta de entrada (anexo 1), realizada a los estudiantes del colegio Alfredo Iriarte.

\begin{tabular}{|c|c|c|c|c|c|}
\hline pregunta & Ideas cuánticas & $\begin{array}{c}\text { Ideas pre- } \\
\text { cuánticas }\end{array}$ & Ideas clásicas & Ideas confusas & N.S / N.R \\
\hline 1 & $0 \%$ & $41 \%$ & $41 \%$ & $18 \%$ & $0 \%$ \\
\hline 2 & $24 \%$ & $30 \%$ & $11 \%$ & $24 \%$ & $11 \%$ \\
\hline 3 & $0 \%$ & $0 \%$ & $6 \%$ & $0 \%$ & $94 \%$ \\
\hline
\end{tabular}

Modelos y representaciones construidas por los estudiantes

Imagen 3. Dibujos hechos por estudiantes diferentes, en los cuales podemos

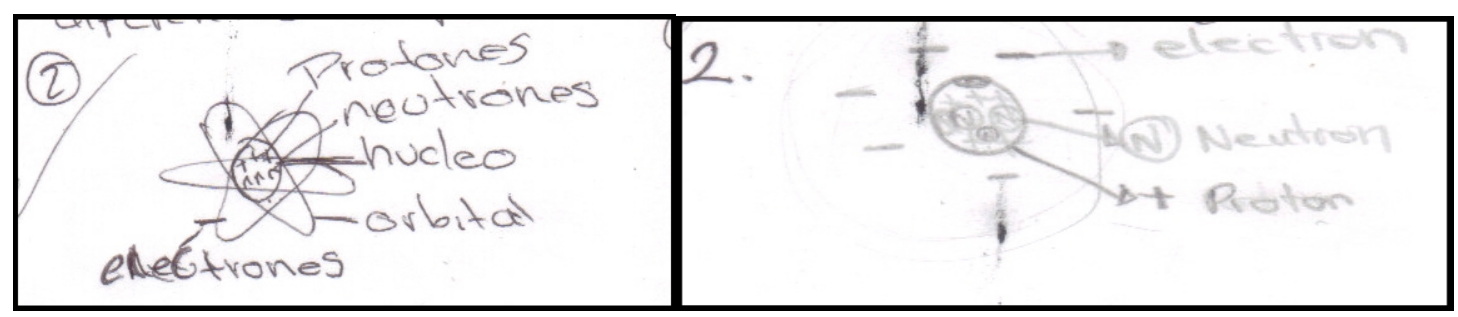


Revista Tecné, Episteme y Didaxis: TED. Año 2014, Número Extraordinario. ISSN Impreso: 0121-3814, ISSN web: 2323-0126

Memorias, Sexto Congreso Internacional sobre Formación de Profesores de Ciencias. 08 al 10 de octubre de 2014, Bogotá

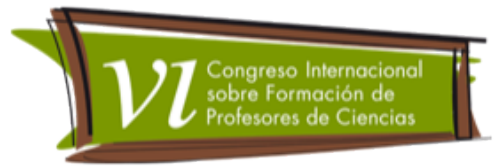

Imagen 4. Representación atómica realizada por los estudiantes del colegio Alfredo Iriarte.

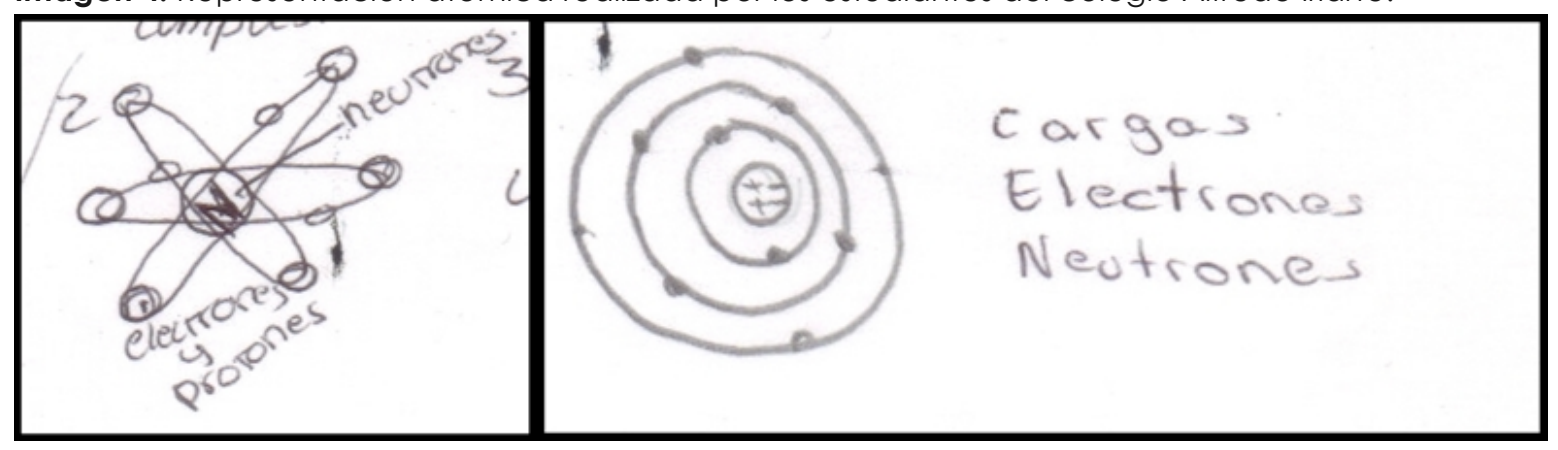

Se continuo con el análisis de la actividad de entrada y se encontró que solo un estudiante de 40 estudiantes, logro responder bien la tercera pregunta la cual consistía en escribir o dibujar las teorías atómicas. Lo que nos muestra que los estudiantes no conocen la historia del átomo.

Finalmente en la pregunta numero 4 decía "¿que se entiende por estado fundamental y estado excitado del átomo?, nos encontramos con respuestas muy variadas dentro de las cuales habían respuestas sin coherencia, permitiéndonos observar que la lectura y la explicación que hicimos, les dejo un concepto muy frágil de lo que era el estado fundamental y excitado del átomo.

Partiendo de los resultados y análisis que obtuvimos en la primera sesión logramos planear y ejecutar la segunda clase aclarando los conceptos que mayor falencias poseían a partir de la temática Auroras Boreales; Se aplicó el ultimo instrumento como evaluación final (tabla 2).

Tabla 2. Resultado de la evaluación final, realizada por los Estudiantes del colegio Alfredo Iriarte.

\begin{tabular}{|c|c|c|c|c|c|}
\hline respuesta & ideas cuánticas & $\begin{array}{c}\text { ideas } \\
\text { pre- } \\
\text { cuánticas }\end{array}$ & ideas clásicas & ideas confusas & n.s / n.r \\
\hline 1 & $21 \%$ & $57 \%$ & $0 \%$ & $21 \%$ & $0 \%$ \\
\hline 2 & $28.5 \%$ & $21 \%$ & $28.5 \%$ & $21 \%$ & $0 \%$ \\
\hline
\end{tabular}

Logramos inicialmente observar que a la pregunta 1, la cual pedía nuevamente que se definiera lo que era átomo, ya habían conceptos construidos desde una perspectiva cuántica, ya se tenía más claridad de que era el átomo.

En el segundo punto se realizó la comparación de los dibujos realizados en la actividad de entrada y en la evaluación, para de esta manera lograr identificar, 
Revista Tecné, Episteme y Didaxis: TED. Año 2014, Número Extraordinario. ISSN Impreso: 0121-3814, ISSN web: 2323-0126

Memorias, Sexto Congreso Internacional sobre Formación de Profesores de Ciencias. 08 al 10 de octubre de 2014, Bogotá

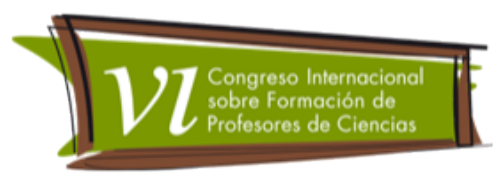

avances, retrocesos, confusiones y los resultados de nuestra labor. Logramos ver claramente que en la mayoría de los casos tuvimos grandes avances, empezando por que los estudiantes ya ubicaban correctamente cada una de las partículas sub-atómicas (imagen 5.a), y al mismo tiempo logramos pasar de la concepción de átomo clásica, se cambia el concepto de orbita por orbital y se piensa en un modelo más cercano al cuántico (Imagen 5b-c).

Imagen 5. A) Dibujos realizados por un estudiante, el primero (izquierda) pertenece a la actividad de entrada, mientras que el segundo (derecha) pertenece a la evaluación final.

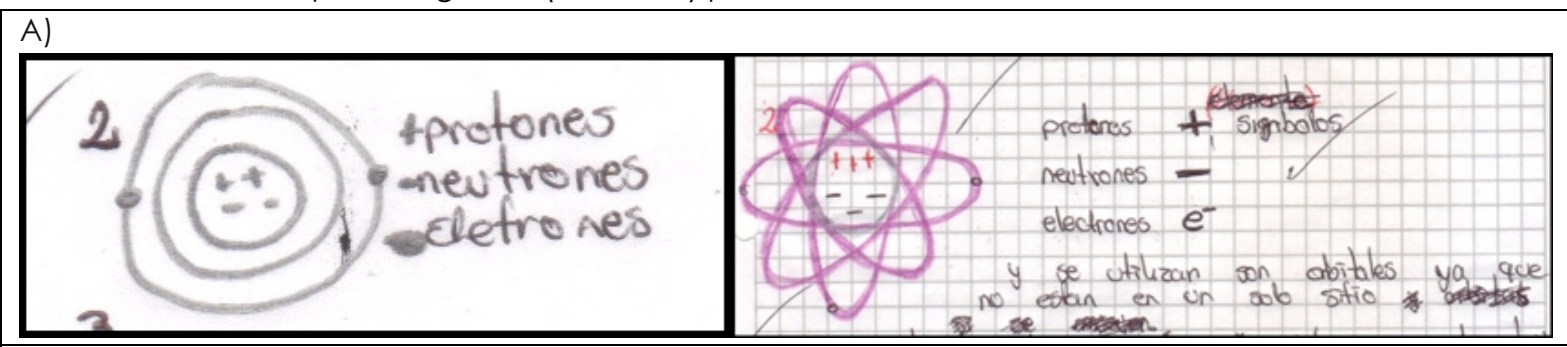

B)

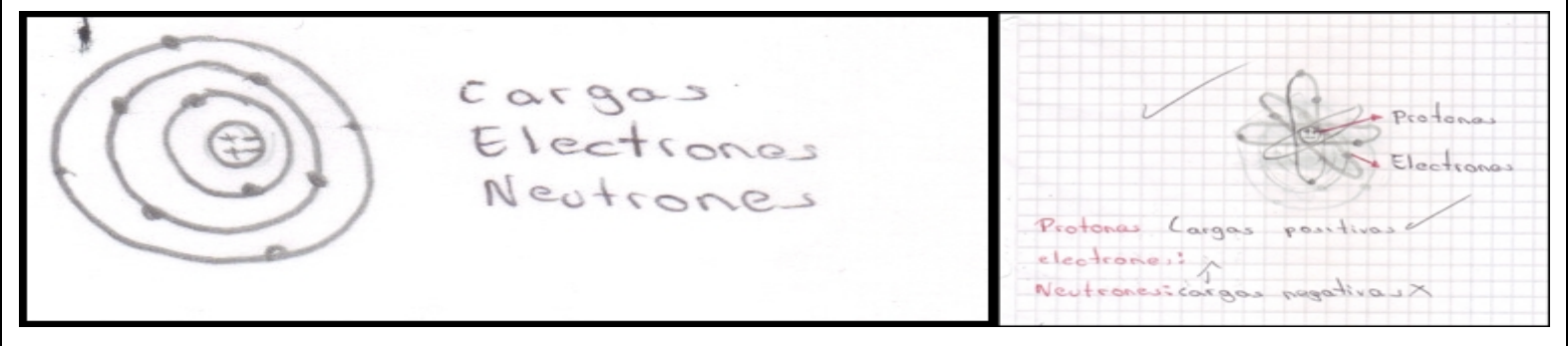

C)

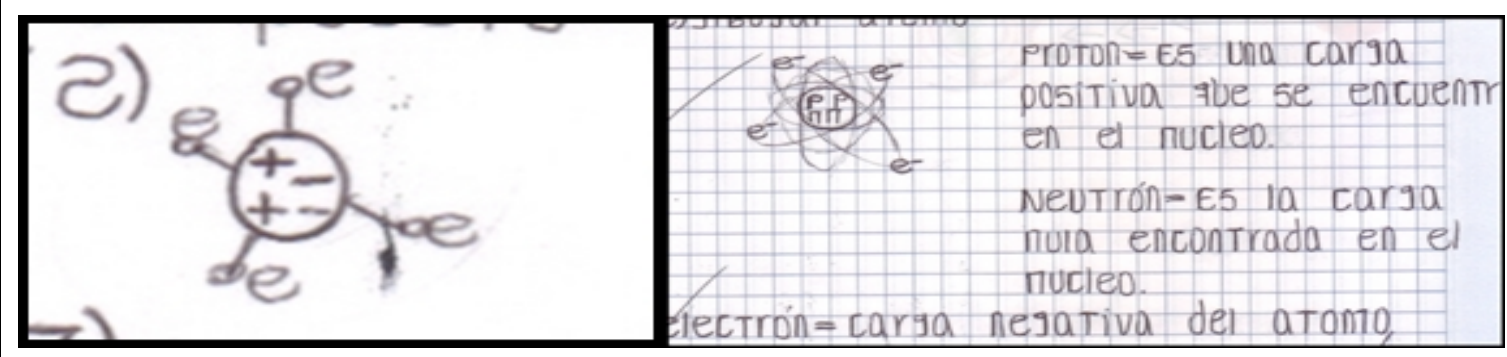

El último punto contemplaba lo más importante, se les solicitaba a los estudiantes realizar un dibujo donde se representara la formación de las "Auroras Boreales", teniendo en cuenta que esta era la temática de la cual partíamos para explicar otros conceptos, debíamos observar si habíamos dejado clara la temática central; y nos encontramos con que los estudiantes dentro de sus escritos y sus 
Revista Tecné, Episteme y Didaxis: TED. Año 2014, Número Extraordinario. ISSN Impreso: 0121-3814, ISSN web: 2323-0126

Memorias, Sexto Congreso Internacional sobre Formación de Profesores de Ciencias. 08 al 10 de octubre de 2014, Bogotá

dibujos eran muy claros al explicar las auroras boreales, tanto que entendían como estas se formaban (Imagen 6).

Imagen 6. A) B) C) Dibujos realizados por 3 estudiantes representando la formación de las "Auroras Boreales".
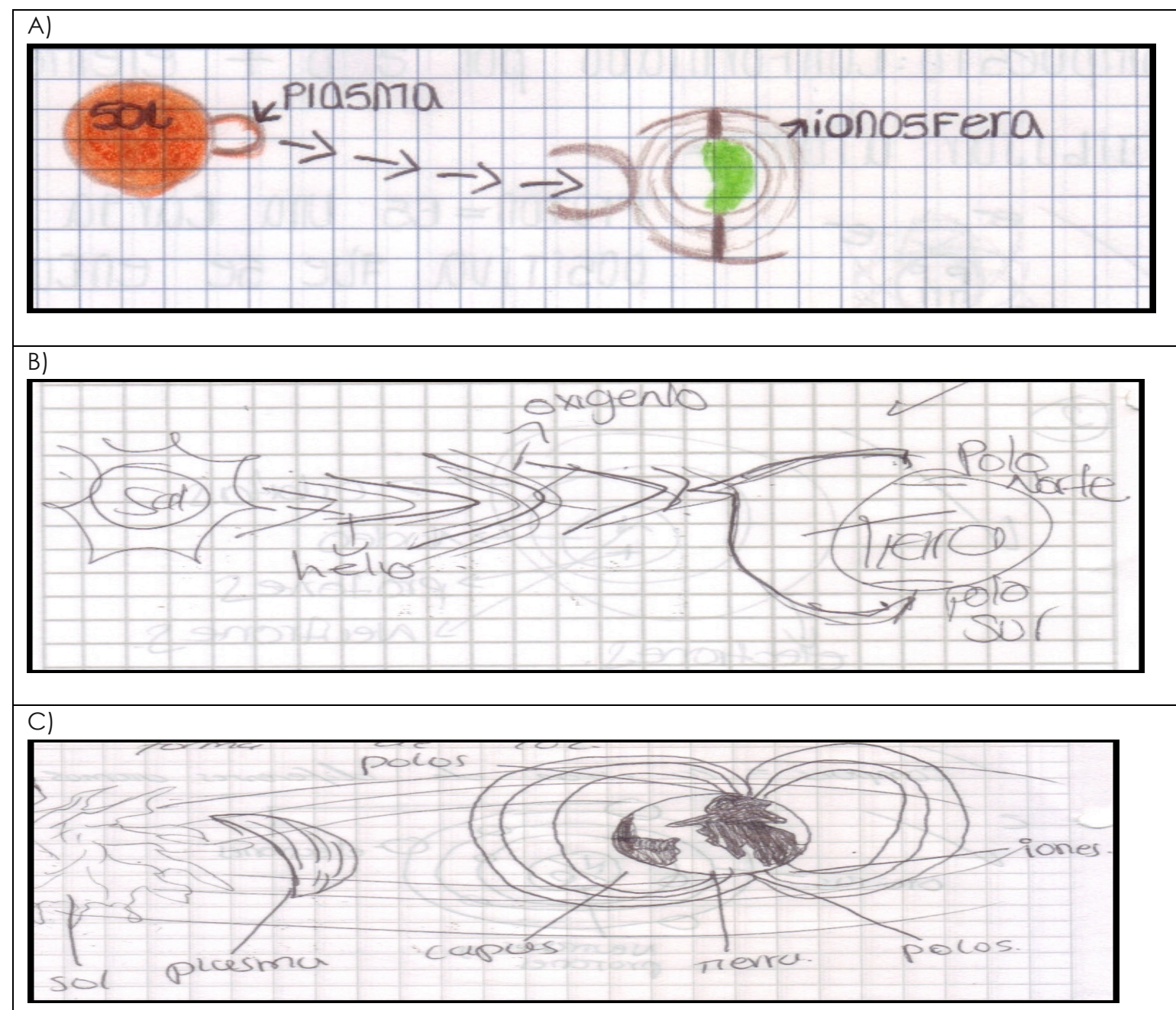

Con respecto a este último punto, los estudiantes demostraron en cada uno de los dibujos la identificación de los contenidos metodológicos - conceptuales de la química, relacionándolos y explicándolos desde fenómenos particulares como las "Auroras Boreales". 
Revista Tecné, Episteme y Didaxis: TED. Año 2014, Número Extraordinario. ISSN Impreso: 0121-3814, ISSN web: 2323-0126

Memorias, Sexto Congreso Internacional sobre Formación de Profesores de Ciencias. 08 al 10 de octubre de 2014, Bogotá

\section{Conclusiones}

Se logró generar en el aula de clases conceptos claros que son claves dentro del proceso de aprendizaje de la química, como: átomo, molécula, elemento, compuesto, ion, entre otros, que fueron acogidos con gran empeño e interés a medida que se iba desarrollando la explicación de la temática: "auroras boreales".

Con los resultados de la evaluación del proceso de aprendizaje Se observó que los estudiantes afianzaron los conceptos claves, especificando de forma clara y precisa todo el conocimiento que habían adquirido a acerca de la temática,

Por otro lado se dejó claro que a partir de la falta de enseñanza que instruye cada uno de los profesores en el aula de clases deja por entendido que todos los estudiantes aprenden de igual forma, generando así una clase en el tablero, sin mayor variación que el color del marcador. Pero y entonces para que hablamos de didáctica de las ciencias o de nuevas corrientes pedagógicas, si al enfrentarnos a un grupo de estudiantes, nos quedamos con el tradicionalismo y dejamos los modelos pedagógicos como una simple palabra que se queda sin innovar, retomar y progresar en nuestras aulas de clases.

\section{Referencias bibliográficas}

Chamizo J.A. 2010. Una tipología de los modelos para la enseñanza de las ciencias. Revista Eureka sobre Enseñanza y Divulgación de las Ciencias, 7 (1) pág. $26-41$.

Ferro, V.R., González-jonte, R. H., Cruz, Z. 1995. Una reflexión curricular sobre la enseñanza de la estructura de la sustancia en la formación de profesores de química. Revista Enseñanza de las Ciencias, 13 (3) pág. 371-377.

Stern, D., Peredo, M. 2000. La aurora polar. Recuperado de http://wwwistp.gsfc.nasa.gov/Education/Mauroral.html

Akasofu, S. 1989. La aurora dinámica. Revista investigación y ciencia. № 154. pág. 42-50. Recuperado de http://www.investigacionyciencia.es/investigacion-y ciencia/numeros/1989/7/la-aurora-dinmica-3047

Pérez, F. F. G. (2000). Los modelos didácticos como instrumento de análisis y de intervención en la realidad educativa. Revista Electrónica de La Universidad de Barcelona, Barcelona, (207). 
Revista Tecné, Episteme y Didaxis: TED. Año 2014, Número Extraordinario. ISSN Impreso: 0121-3814, ISSN web: 2323-0126

Memorias, Sexto Congreso Internacional sobre Formación de Profesores de Ciencias. 08 al 10 de octubre de 2014, Bogotá

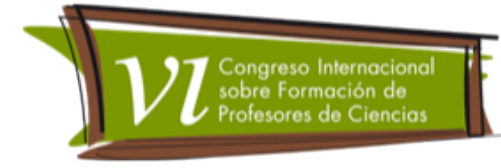

Bloxham J., Gubbins D. (1990). La evolución del campo magnético terrestre. Revista mundo científico. No161. Recuperado de http://www.nationalgeographic.es/medio-ambiente/el-campo-magn

Como se origina una aurora boreal. Youtube enlace. 2011. Recuperado de http://youtu.be/L_k92H7KQAg 